\title{
Photovoltaic Devices Based on Photo Induced Charge Transfer in Polythiophene:CN-PPV Blends
}

\author{
L. S. Roman ${ }^{a *}$ A. C. $\operatorname{Arias}^{b}$, M. Theander ${ }^{a}$, M. R. Andersson ${ }^{c}$ and O. Inganäs ${ }^{a}$ \\ ${ }^{a}$ Laboratory of Applied Physics, Dept. of Physics, Linköping University, Linköping, S-58183, Sweden \\ ${ }^{b}$ Cavendish Laboratory Madingley Road Cambridge CB3 OHE, United Kingdom \\ ${ }^{c}$ Dept. of Organic Chemistry and Polymer Technology, Chalmers University of Technology, S-412 96 Göteborg, Sweden
}

Received on 6 January, 2003

\begin{abstract}
We have investigated the photovoltaic properties of polymer devices based on a blend of two polymers, (poly(3-(2'-methoxy-5'-octylphenyl) thiophene) (POMeOPT) and poly(2,5,2',5'-tetrahexyloxy-7,8'-dicyanodi-p-phenylenevinylene) (CN-PPV), where photo induced charge transfer takes place at the interfaces formed by the interpenetrating network of the donor and the acceptor. The polymer blend films resulting from the spin coating were phase separated and the photo response of the devices depended on the morphology of the segregation. We have studied different blend ratios by photoluminescence quenching, scanning force microscopy and photovoltaic measurements. External quantum efficiencies of $4.5 \%$ at $500 \mathrm{~nm}$ were achieved for the less segregated polymer blend.
\end{abstract}

\section{Introduction}

The possibility in using the photovoltaic effect for energy conversion has been motivated the research on this field along the years. The focus was mostly in the use of inorganic materials. Recently, with the use of photo induced charge transfer across the internal or external donor-acceptor (D/A) heterojunctions, [1] the organic photovoltaic devices have been demonstrated to be highly efficient, attracting attention. [2-10] Organic materials in general and polymers in particular have been extensively studied due to the advantages these materials present when compared to inorganic semiconductors. Polymers are easily spin-coated from solution and do not require ultra-clean environment during any processing procedure. In addition, these materials can be chemically tuned so that their absorption and emission color covers the entire visible spectrum. [11] High quality polymers are being synthesized with high degree of reproducibility, which leads to a great development in stability, lifetimes and performance in all areas of application.

The use of polymer blends as the active layer in optoelectronic devices is an alternative to change and improve the device characteristics and performance. LEDs in which the emission color varies as a function of the applied voltage were made by blending conjugated polymers that have different emission spectrum. [12] Efficient photodiodes have been fabricated from mixtures of electron-accepting materials and hole-accepting polymers. [2-10] The photo gen- erated excitons are dissociated at the interfaces, which are dispersed in the bulk of the blend film. Charges are transported through the respective phase, driven by the internal electric field.

The photovoltaic properties of $\mathrm{CN}-\mathrm{PPV} / \mathrm{POMeOPT}$ blends have been investigated in this work, where (poly(3(2'-methoxy-5'-octylphenyl) thiophene) (POMeOPT) $[11,14]$ was the "donor" and poly $(2,5,2$ ',5'-tetrahexyloxy7,8'-dicyano-di-p-phenylenevinylene) (CN-PPV), [5] was used as the electron-accepting material. The chemical structures of the polymers are presented in Fig. 1. The blend was investigated by comparing morphology, photoluminescence and action spectra of several compositions.

\section{Experimental}

The photovoltaic devices were fabricated in a sandwich structure starting with the hole collector electrode, a transparent layer of PEDOT(PSS) (poly(3,4EthyleneDioxyThiophene) (Baytron - Bayer AG) doped with polystyrenesulfonate) deposited by spin coating onto Indium Tin Oxide (ITO)/Glass substrate, from a water dispersion solution, with the thickness 80nm. The PEDOT(PSS) was patterned in order to cover only ITO. PEDOT(PSS) is used as electrode because of its good injection/collection properties. The active part of the photodiode was a blend of two photoactive semiconducting polymers

\footnotetext{
*Present address: Department of Physics, Federal University of Paraná, Curitiba, Brazil. 81531-990.
} 


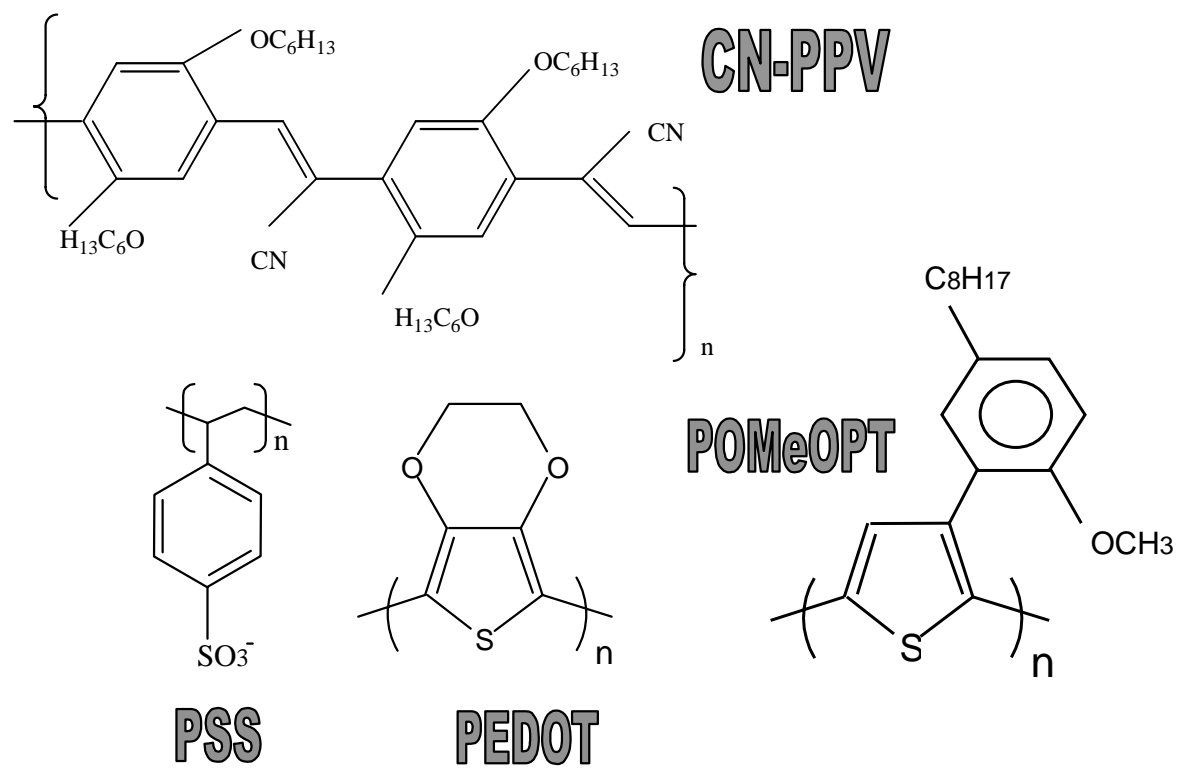

Figure 1. Chemical structure of the polymers used in the photodiodes. Neat POMeOPT, CN-PPV, and their mixtures were the active layer and PEDOT doped with PSS was the anode.

$\mathrm{CN}-\mathrm{PPV}$ and POMeOPT. The polymer blend was deposited by spin coating onto PEDOT(PSS)/ITO electrode, from a chloroform solution with different polymer ratios (1:9), $(1: 1)$ and $(9: 1)$, with the thickness $60 \mathrm{~nm}$, an exception is made for the blend (9:1) that was quite rough being difficult to estimate its thickness. All thicknesses were measured with DEkTAK surface profilometer. The electron collector electrode, Aluminum, was evaporated onto the blend layer through a shadow mask defining the active area for the devices, from 6 to $10 \mathrm{~mm}^{2}$.

The photoluminescence (PL) quantum yield measurements were performed in an integrating sphere described in detail in ref. [13]. Polymer mixtures were made by making chloroform solutions of each polymer $(5 \mathrm{mg} / \mathrm{ml})$ and mixing the respective solution with ratios 1:9, 1:1 and 9:1 and spinning at $1000 \mathrm{rpm}$ on top of quartz substrates. Samples were excited at $495 \mathrm{~nm}$, close to the absorption maximum for both POMeOPT (530 nm) and CN-PPV (502 nm).

The absorption spectra were taken with a Perkin Elmer $\lambda 9$ spectrometer. The action spectra were taken using a Keithley 485 picoammeter to measure the photocurrent under incidence of monochromatic light through ITO or Al side. The light was from Oriel MS257 monochromator using a Tungsten Halogen lamp. The current versus voltage characteristics was measured with a Keithley 237 electrometer under dark and under monochromatic illumination of certain wavelengths. Experiments of photocurrent dependence on light intensity were done using fused silica gray filters in the light path. The light intensity was measured using a calibrated Si photodiode at the sample position.

\section{Results and discussion}

The normalized absorption spectra of spin coated films of POMeOPT and CN-PPV neat and the blends at different compositions are presented in Fig. 2. It is possible to notice that the major part of CN-PPV and POMeOPT spectra overlap, CN-PPV has the peak for the absorption 502nm and POMeOPT in 530nm, with major part of their spectra overlapping.

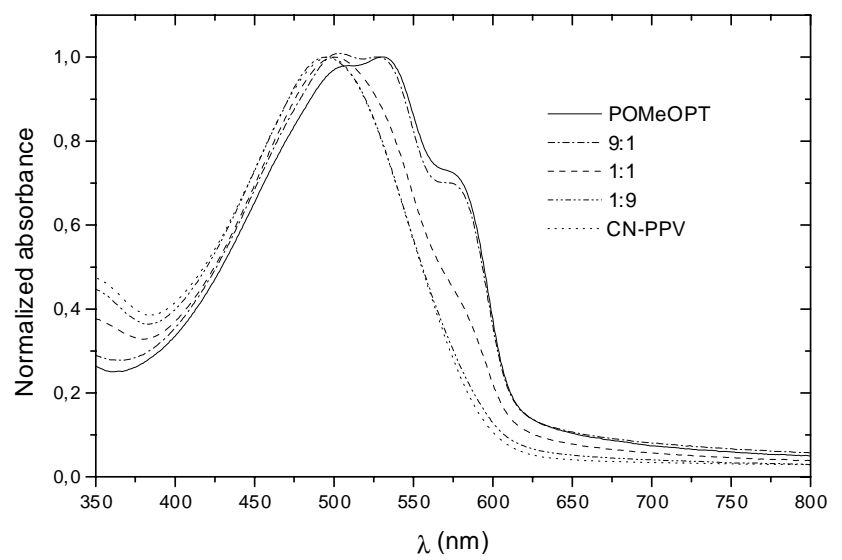

Figure 2. Normalized absorption spectra of spin coated films of POMeOPT, POMeOPT:CN-PPV (9:1), (1:1), (1:9), and CN-PPV.

The Fig. 3 shows the photoluminescence (PL) quantum yield of the polymer blends as a function of the weight ratio of the polymers in the solution used for spin coating the films. The photoluminescence quenching is one technique used as indicative of electron transfer from one organic material to the other, where the materials present photoluminescence of few percent. The PL quenching is moderate compared to other mixture described in ref. [5]. The inter- 
pretation of the data is difficult as the absorption and emission spectra of the two polymers overlap extensively. The influence of excitation transfer may therefore be hard to distinguish from that of electron transfer. The electron transfer should however be visible as a photocurrent in photovoltaic device. In order to compare the photovoltaic devices fabricated using these blends, spectral response from photodiodes with active layer of neat polymers and the blend POMeOPT:CN-PPV in the ratio (9:1), (1:1), and (1:9) were taken. To facilitate, the different combinations for the polymers blends will be referred depending on the percent of CN-PPV in the blend, $10 \%, 50 \%$, or $90 \%$.

When blending these two polymers phase segregation occurred. The morphology of the spincoated films of POMeOPT:CN-PPV blends onto ITO/PEDOT(PSS) substrate is presented in the SFM pictures in Fig. 4. The reference measurements have shown that the surfaces of the neat polymers imaged with SFM were smooth.

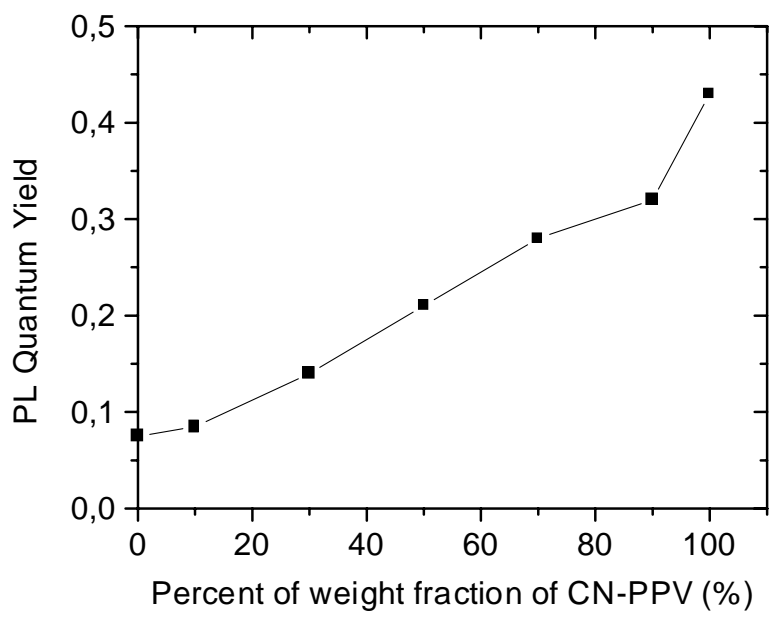

Figure 3. Photoluminescence quantum yield of the neat POMeOPT and CN-PPV polymers and their mixture regarding the percent of weight fraction of CN-PPV in the blend.

The pictures were taken in height and phase simultaneously scanned. The phase mode gives information about the stiffness of the polymer, making it possible to obtain more contrast between the polymer phases. The morphology of the segregated polymer blend plays an important part for the charge transfer possibilities. The surface of the $90 \%$ blend film was quite rough. The other two combinations presented smoother surface and, comparing their morphology though the SFM images of the $10 \%$ and $50 \%$ mixtures, it is possible to notice that the $10 \%$ blend film shows less segregation than the $50 \%$ blend film. In order to compare the morphology with the photoresponse for each blend, in Fig. 5 is presented the short circuit action spectra of the ITO/PEDOT/blend/Al devices. The action spectra is given by the incident monochromatic photon to current conversion efficiency (IPCE) that is obtained by measuring the short circuit photocurrent of the device while the device is illuminated by monochromatic light at different wavelengths.
The spectral response of neat polymer photodiodes presented quite small IPCE\%, at absorption peak position for POMeOPT the IPCE\% was $1.5 \times 10^{-3} \%$ and for CN-PPV, IPCE\% was $7 \times 10^{-4} \%$. The action spectra of these photodiodes followed well the absorption profile in both cases while illuminated from ITO/PEDOT(PSS) or Al electrodes. The efficiency of the blend diodes increased drastically compared to the neat diodes, suggesting that charge transfer occur between the polymers. The spectral responses of the photodiodes were taken with illumination through ITO/PEDOT and Al electrodes presenting quite similar spectral shapes and following the absorption spectra of the respective blend. An exception was found for the spectrum taken from $90 \%$ blend through $\mathrm{Al}$ electrode where the relative contribution from POMeOPT was higher. CN-PPV has quite high molecular weight and in studies of another set of polymer blends it was suggested that due to the high molecular weight of CN-PPV this polymer would deposit first during the spin coating process leaving a stratified structure. [15] In our studies we found the topological morphology of $90 \%$ blend quite rough and the spectral response of $90 \%$ blend diodes distinctly depending on the illuminated electrode. It may suggest that the $90 \%$ blend forms stratified layers where the second layer (POMeOPT) is not continuous, this would be in agreement with the fact that under illumination through $\mathrm{Al}$ electrode the contribution from POMeOPT phase is higher relative to $\mathrm{CN}-\mathrm{PPV}$.

The highest external quantum efficiency was found for the $10 \%$ blend, about $4.5 \%$ at $\lambda=500 \mathrm{~nm}$. The opposite mixture, $90 \%$ blend, presented rather poor efficiency, $0.5 \%$. These photoresponse results were already suggested from the blend morphologies experiments, where smaller segregation phases leads to increased number of dispersed interfaces suitable for the exciton ionization. In addition the photoluminescence experiments suggested that the $10 \%$ and $90 \%$ blends presented higher PL quenching than the $50 \%$ blend.

Polymer blends can change their morphology upon annealing, following the spin coating the samples were annealed up to $60^{\circ} \mathrm{C}$ during 1 minute. After that, the $\mathrm{Al}$ electrode was deposited by evaporation. The topological morphology of the $10 \%$ and $50 \%$ blends were quite similar to their morphology before the annealing treatment, but the photoconversion efficiency decreased, most likely due to the polymer oxidation than morphology. The efficiency for the $10 \%$ and $50 \%$ blends after annealing at $\lambda=500 \mathrm{~nm}$ were $2.7 \%$ and $0.9 \%$, respectively. For the $90 \%$ blend the film become smooth with a low level of phase segregation but the photoconversion efficiency maintained its low numbers, $0.5 \%$.

Current density - voltage characteristics in dark and under illumination $\left(500 \mathrm{~nm}\right.$ wavelength with $2,25 \mathrm{~W} / \mathrm{m}^{2}$ light intensity) of the $10 \%$ and $50 \%$ blend devices are pre- 
sented in Fig. 6.
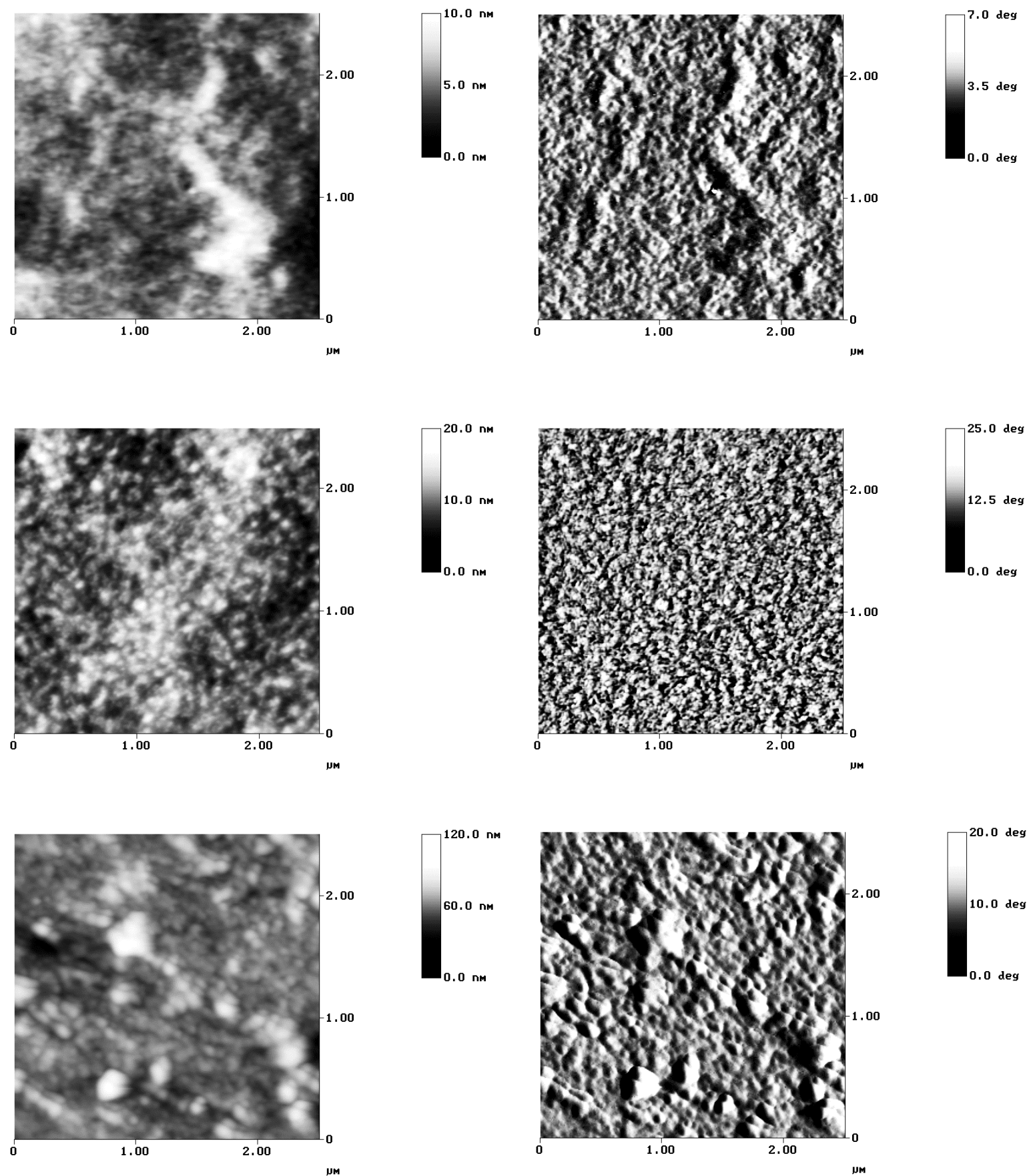

Figure 4. Scanning Force Microscopy images of polymer blend films of POMeOPT:CN-PPV, 10\%(a), 50\%(b), and 90\%(c). Two modes were detected simultaneously during the scan height (left) and phase (right).

The $\mathrm{J}-\mathrm{V}$ characteristics of the $90 \%$ blend in dark and under illumination were almost symmetric with quite poor rectification similar to single layer device of CN-PPV, the current behavior in this blend is ruled by CN-PPV polymer, the major phase in the blend. The open circuit voltage $\left(\mathrm{V}_{o c}\right)$ of $10 \%$ and $50 \%$ blend devices are quite high be- ing $1 \mathrm{~V}$ and $0.9 \mathrm{~V}$, respectively. The fill factor is given by, $F F=\frac{(J V)_{\max }}{J_{s c} V_{o c}}$ where $J$ and $V$ are the values for the current density and voltage for maximizing their product and $\mathbf{J}_{s c}$ is the short circuit current density. The fill factors of $10 \%$ and $50 \%$ blend devices are found to be 0.13 and 0.2 , respectively. 


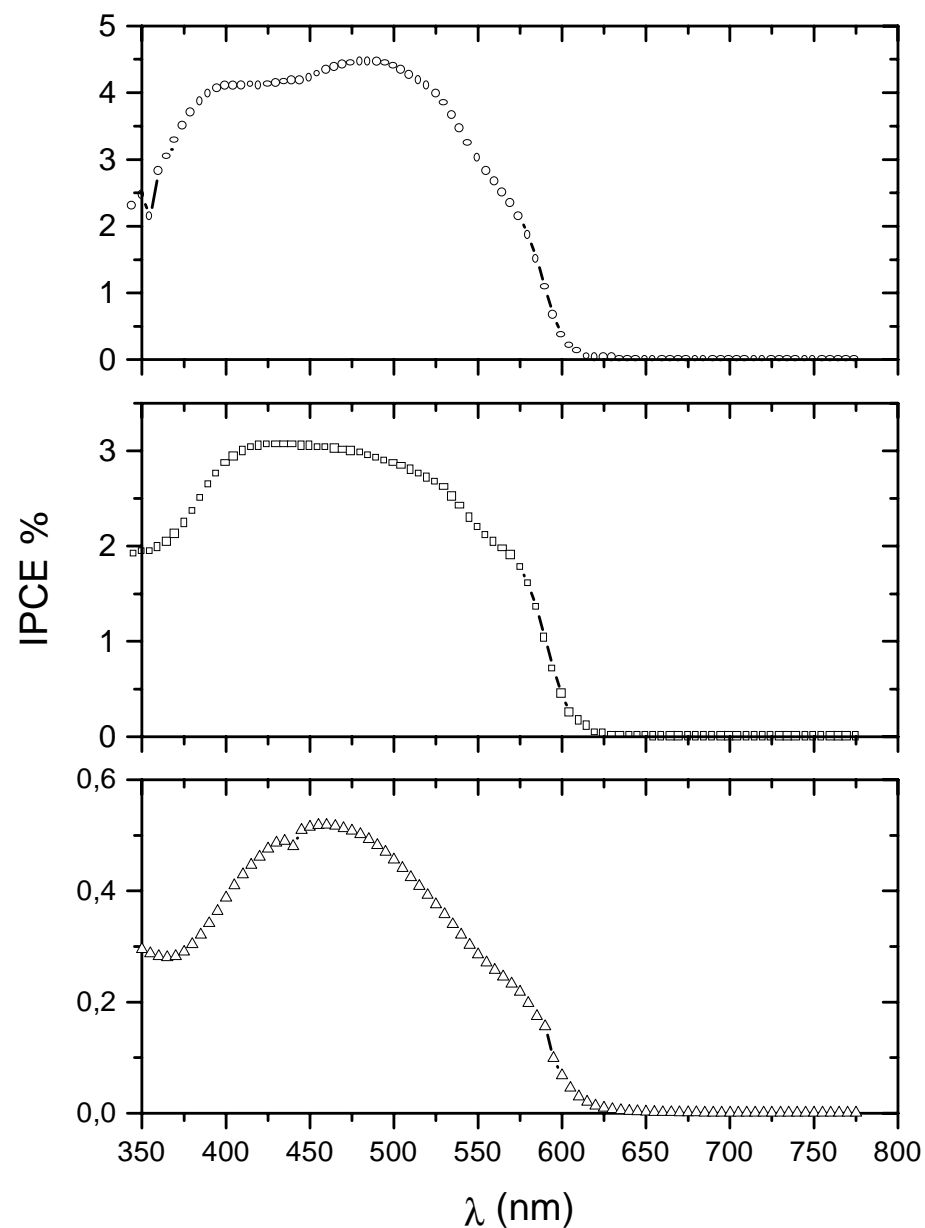

Figure 5. Short circuit spectral response of ITO/PEDOT(PSS)/ blend/Al devices for the blend POMeOPT:CN-PPV, (10\%) (circles), (50\%) (squares), and (90\%) (triangles). The photodiodes were illuminated from ITO/PEDOT(PSS) electrode.

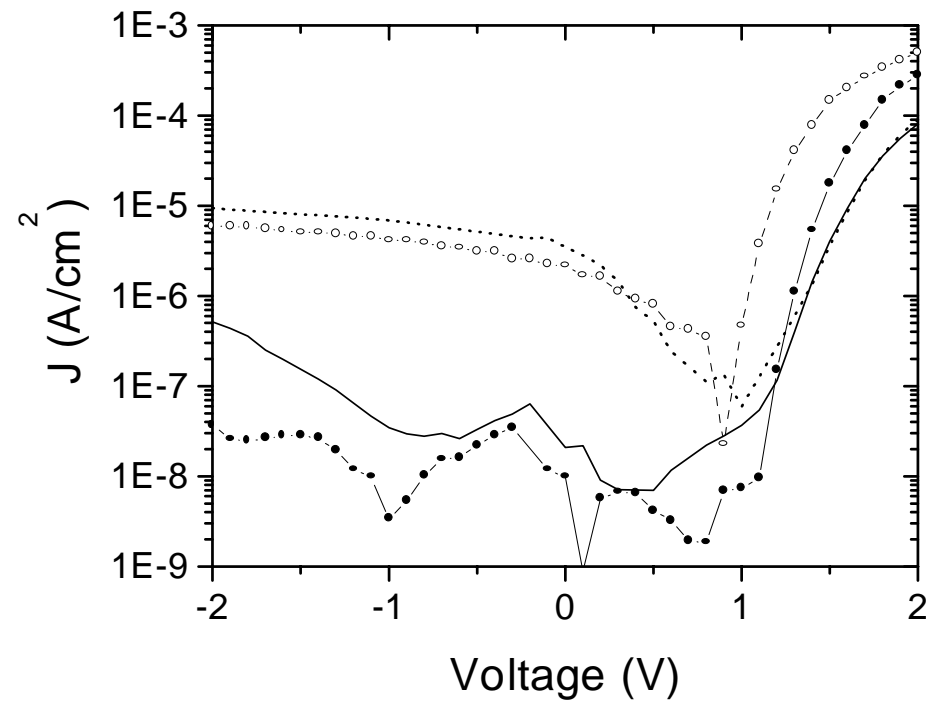

Figure 6. Current density versus applied voltage characteristics of the ITO/PEDOT(PSS)/blend/Al devices, in dark and under illumination at the $500 \mathrm{~nm}$ wavelength with $2,25 \mathrm{~W} / \mathrm{m}^{2}$ light intensity. The blends used were POMeOPT:CN-PPV (9:1), 10\% solid line/dark and dashed line/light, and POMeOPT:CN-PPV (1:1), 50\% solid circles/dark and open circles/light. 


\section{Conclusions}

We have investigated photovoltaic properties of devices based on polymer blends active layers where photoinduced charge transfer took place. Different combinations of POMeOPT and CN-PPV polymers formed the blends. We have correlated the photoconversion efficiency with topological morphology and photoluminescence quenching. The quenching of emission may be a good indicative for charge transfer but not enough since the photocurrent is dependent on the transport of the photogenerated charges to the electrodes, which depends on the blend morphology. Photodiode prepared from the less segregated polymer blend presented external quantum efficiencies as high as $4.5 \%$ at $\lambda=500 \mathrm{~nm}$.

\section{Acknowledgements}

The authors are grateful to F. Cacialli for providing the CN-PPV polymer used in this work. A.C.A. acknowledges the scholarship from $\mathrm{CNPq}$ Brazilian science foundation. The Göran Gustafsson foundation, the Carl Trygger Foundation and the Swedish Research Council for Engineering Sciences (TFR) supported this work.

\section{References}

[1] N. S. Sariciftci, and A. J. Heeger, in Handbook of conductive molecules and polymers; Vol. 1, edited by H. S. Nalwa (John Wiley\&Sons, 1997).

[2] N. S. Sariciftci, D. Braun, C. Zhang, V. I. Srdanov, A. J.
Heeger, G. Stucky, and F. Wuld, Appl. Phys. Lett. 62, 585 (1993).

[3] G. Yu, J. Gao, J. C. Hummelen, F. Wudl, and A. J. Heeger, Science 270, 1789 (1995).

[4] G. Yu, A. J. Heeger, J. Appl. Phys. 78, 4510 (1995).

[5] J. J. M. Halls, C. A. Walsh, N. C. Greenham, E. A. Marseglia, R. H. Friend, S. C. Moratti, and A. B. Holmes, Nature 376, 498 (1995).

[6] L. S. Roman, M. R. Andersson, T. Yohannes, O. Inganäs, Advanced Materials 9, 1164 (1997).

[7] L. S. Roman, W. Mammo, L. A. A. Pettersson, M. Andersson, and O.Inganäs, Adv. Materials 10, 774-777 (1998).

[8] M. Granström, K. Petrisch, A. C. Arias, A. Lux, M. R. Andersson, and R. H. Friend, Nature 395, 257-260 (1998).

[9] S.E. Shaheen, C. J. Brabec, N. S. Sariciftici, E. Padinger, T. Framherz, J. C. Hummelen, Appl. Phys. Lett., 78, 841 (2001).

[10] M. Berggren, O. Inganäs, G. Gustafsson, J.Rasmusson, M. R. Anderson, T. Hjertberg, and O. Wennerström, Nature 372, 444 (1994).

[11] M. R. Andersson, W. Mammo, T. Olinga, M. Svensson, M. Theander, O. Inganäs, Synthetic Metals 101, 11 (1999).

[12] R. H. Friend, R. W. Gymer, A. B. Holmes, J. H. Burroughes, R. N. Marks, C. Taliani, D. D. C. Bradley, D. A. dos Santos, J. L. Brédas, M. Lögdlund, W. R. Salaneck, Nature 397, 121 (1999).

[13] M. Theander, O. Inganäs, W. Mammo, T. Olinga, M. Svensson, M. R. Andersson, Journal-of-Physical-Chemistry-B 103, 7771 (1999).

[14] L. S. Roman and O. Inganäs, Synthetic Metals, 125, 419 (2002).

[15] J. J. Halls, Thesis, Cambridge University, 1997. 